\title{
Vendor Record Documentation
}

National Cancer Institute

\section{Source}

National Cancer Institute. Vendor Record Documentation. NCI Thesaurus. Code

C115745.

Records detailing the relationship between a vendor and a clinical trial sponsor, or contract research organization (CRO). 